\title{
Effective Channel Order Determination Algorithm for Convolutive Blind Channel Identification
}

\author{
Senquan Yang ${ }^{1}$, Haifeng $\mathrm{Su}^{2}, \mathrm{Pu} \mathrm{Li}^{1}{ }^{1}$, Songxi Hu${ }^{1}$, Jinru Chen ${ }^{1}$ \\ ${ }^{\prime}$ School of Physics and Electromechanical Engineering, Shaoguan University, Daxue Road, Shaoguan, 512005, China \\ ${ }^{2}$ Department of Machinery and Electronics, Shijiazhuang University of Applied Technology, Shijiazhuang, 050081, China
}

A RT I C LE I N F O
Article History
Received 16 Apr 2019
Accepted 12 Aug 2019
Keywords
Effective channel order
determination
Numerical analysis arguments
Blind channel identification
Higher-order cumulant tensor

\section{INTRODUCTION}

It is well known that a key issue is the determination of effective channel order in convolutive blind channel identification [1-3]. A common assumption in some papers [4,5] is that the effective channel order is known. However, in practice, it is unknown and has an important effect on the channel identification. Especially, in the underdetermined convolutive mixture model [6-8], it is a difficult issue due to the complexity of the model. Therefore, the effective channel order determination is a challenging problem to be solved in the blind channel identification.

Among numerous methods, the information theoretic criteria have become the classical techniques $[9,10]$, such as Bayesian information criterion (BIC) [11], generalized information criterion [12], the model selection introduced by Akaike information criterion (AIC) [13], by Schwartz and Rissanen minimum description length (MDL) $[14,15]$, a method with the random matrix theory (RMT) algorithm [16], the automatic order selection [17], N-way probabilistic clustering $[18,19]$. In addition, an effective method has been proposed based on numerical analysis arguments [20,21], which detects the effective channel order using a rank detection method to the single-input/multiple-output (SIMO) case. By using the concept of standard angle between subspace and invariant subspace perturbation results, the maximum stable decomposition from the range space of data covariance matrix to signal and noise subspace is provided. Moreover, the signal order estimation is also required in several applications of signal processing. For example, signal

\footnotetext{
Corresponding author.Email: lipu_300@sgu.edu.cn
}

rank detection problem in cognitive radio and the source number determination in blind unmixing problems. However, these methods are just suit for the overdetermined case. For the underdetermined case, they have some limitations which may cause inaccuracy of the effective channel order estimation.

To compensate for previous shortcomings, the higher-order cumulant tensor methods [22-24] are capable of processing the underdetermined case, and blindly identify the steering vectors of up to $N^{2}-N+1$ sources from $N$ sensors. Additionally, existing multilinear detection algorithms $[25,26]$ rely on the higher-order singular value decomposition, which are to rearrange the tensor as a matrix by use of an unfolding operation, utilizing the eigenvalues of the resultant matrices to detect the model order. For this reason, higher-order cumulant tensor method has an advantage to deal with the model order selection in the underdetermined case $[27,28]$.

In this paper, an improved algorithm is proposed by combining numerical analysis arguments and higher-order cumulant tensor to deal with the detection problem of effective channel order in the convolutive blind channel identification. First, we provide a straightforward theoretical analysis to illustrate the effectiveness of the proposed algorithm. Especially, in the SIMO case, the improved algorithm directly provides the effective channel order determination. Second, for the underdetermined convolutive case, supposing the number of source signals is known, we exploit the information contained in the higher-order data statistics and rearrange the tensor as a matrix by using an unfolding operation, then utilize the eigenvalues of the matrix and combine numerical analysis arguments to estimate the effective channel order. 
The contribution of this paper are as follows.

1. We propose an improved algorithm by integrating the merit of higher-order cumulant tensor and numerical analysis arguments to estimate the effective channel order for convolutive mixture model.

2. We employ higher-order cumulant tensor to solve the source number estimation for underdertermined linear mixture case. Meanwhile, the proposed algorithm can be directly used to detect the effective channel order for the SIMO convolutive mixture case. Experimental results demonstrate the effectiveness and superiority of the proposed algorithm.

The structure of the remaining of this paper is organized as follows. The system model statement and algorithm overview is presented in Section 2. Section 3 introduces the numerical analysis arguments and higher-order cumulant tensors. Experiment results are shown in Section 4. Finally, Section 5 summarizes the conclusion and future work.

Notations: In this paper, $N$ denotes the number of microphones, $K$ denotes the number of source signals, $L$ denotes the effective channel order, I denotes the identity matrix, * denotes the linear convolutive operator, $\otimes$ denotes the Kronecker product, $\odot$ denotes the Khatri-Rao product, $(\cdot)^{T}$ denotes the transpose, $(\cdot)^{*}$ denotes the complex conjugate, $(\cdot)^{H}$ denotes the Hermitian transpose, and $E[\cdot]$ denotes the expectation operator.

\section{SYSTEM MODEL AND ALGORITHM OVERVIEW}

\subsection{Convolutive Model}

Consider $N$ sensors and $K$ source signals $(N<K)$, the system model employed in this paper can be expressed as

$$
\mathbf{x}(t)=\mathbf{A} * \mathbf{s}(t)+\mathbf{n}(t)=\sum_{l=0}^{L-1} \mathbf{A}(l) \mathbf{s}(t-l)+\mathbf{n}(t)
$$

where $\mathbf{s}(t)=\left[s_{1}(t), \ldots, s_{K}(t)\right]^{T}$ represents the source signals, $\mathbf{x}(t)=\left[x_{1}(t), \ldots, x_{N}(t)\right]^{T}$ denotes the observed mixture signals, $\mathbf{A}(l)$ is the mixing system at time-lag $l, \mathbf{n}(t)=\left[n_{1}(t), \ldots, n_{N}(t)\right]^{T}$ is the additional noise. The main objective of this paper is to detect the effective channel order $L$.

\subsection{Algorithm Overview}

We propose an improved algorithm to detect the effective channel order for convolutive blind channel identification. The block diagram of the proposed algorithm is shown in Figure 1. The processing is based on two-step method. In the first step, we exploit the information contained in the higher-order data statistics and rearrange the tensor as a matrix using the unfolding operation. Then we estimate the covariance matrix of the data, and compute the eigenvalues of the covariance matrix using eigenvalue decomposition. In the second step, we detect the effective channel order using numerical analysis arguments.

\section{NUMERICAL ANALYSIS ARGUMENTS AND HIGH-ORDER CUMULANT TENSOR}

\subsection{Numerical Analysis Arguments}

In the following, we consider the one-input/two-output case, the results can be extended to the multiple-input/multiple-output (MIMO) situation. We denote the impulse response of the $n$th channel, $n=1,2$, by $\mathbf{a}_{L}^{n}=\left[a_{o}^{n}, \cdots, a_{L-1}^{n}\right]^{T}$, and the entire channel response is denoted by $\mathbf{a}_{L}=\left[\mathbf{a}_{L}^{1}, \mathbf{a}_{L}^{2}\right]^{T}$. Then the convolution matrix $\mathcal{H}\left(\mathbf{a}_{L}\right)$ is expressed as

$$
\mathcal{H}\left(\mathbf{a}_{L}\right)=\left[\begin{array}{c}
\mathcal{F}\left(\mathbf{a}_{L}^{1}\right) \\
\mathcal{F}\left(\mathbf{a}_{L}^{2}\right)
\end{array}\right]
$$

where $\mathcal{F}\left(\mathbf{a}_{L}^{n}\right)$ is denoted as

$$
\mathcal{F}\left(\mathbf{a}_{L}^{n}\right)=\left[\begin{array}{cccccc}
a_{0}^{n} & \cdots & \cdots & a_{L}^{n} & & \\
& \ddots & & & \ddots & \\
& & a_{0}^{n} & \cdots & \cdots & a_{L}^{n}
\end{array}\right]
$$

The additive channel noise is assumed with zero mean and unit variance, i.e.,

$$
\mathbf{E}\left[\mathbf{n}(t) \mathbf{n}^{H}(t)\right]=\sigma^{2} \mathbf{I}
$$

Therefore, the covariance matrix of $\mathbf{x}(t)$ is expressed as

$$
\begin{aligned}
\mathbf{R}_{\mathbf{x}} & =E\left[\mathbf{x}(t) \mathbf{x}^{H}(t)\right]=\mathcal{H}\left(\mathbf{a}_{L}\right) \mathcal{H}^{H}\left(\mathbf{a}_{L}\right)+\sigma^{2} \mathbf{I} \\
& =\mathbf{R}_{\mathbf{L}}+\mathbf{E}_{\mathbf{L}}
\end{aligned}
$$

where $\mathbf{R}_{\mathbf{L}}$ denotes the covariance matrix associated with significant part of the channel, $\mathbf{E}_{\mathbf{L}}$ represents the influence of the additive channel noise, and the influence of the estimated inexact statistics. It is assumed that $\mathbf{E}_{\mathbf{L}}$ is small with respect to $\mathbf{R}_{\mathbf{L}}$.

Denoting the eigenvalues of $\mathbf{R}_{\mathbf{L}}$ as

$$
\begin{aligned}
& \lambda_{1}\left(\mathbf{R}_{\mathbf{L}}\right) \geq \cdots \geq \lambda_{K}\left(\mathbf{R}_{\mathbf{L}}\right)> \\
& \lambda_{K+1}\left(\mathbf{R}_{\mathbf{L}}\right)=\lambda_{K+2}\left(\mathbf{R}_{\mathbf{L}}\right)=\cdots=\lambda_{N}\left(\mathbf{R}_{\mathbf{L}}\right)=\sigma^{2}
\end{aligned}
$$

The distance measure between two linear subspaces commonly used for numerical analysis is the sine of their standard angles.

$$
\begin{aligned}
T & \doteq\left\|\sin \angle\left(R\left(\hat{S}_{K}\right), R\left(S_{K}\right)\right)\right\|_{2} \\
& \leq \frac{\left\|\varepsilon_{L}\right\|_{2}+\lambda_{K+1} T}{\hat{\lambda}_{K}}=\frac{\lambda_{K+1}+\lambda_{K+1} T}{\lambda_{K+1}+\delta}
\end{aligned}
$$

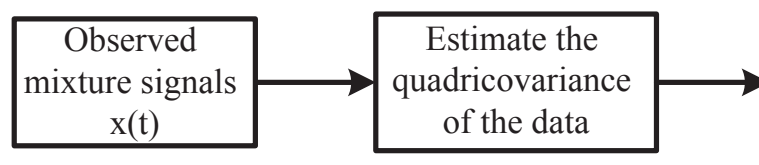

$$
\begin{aligned}
& \text { Compute the } \\
& \text { eigenvalues of } \\
& \text { the matrix }
\end{aligned}
$$

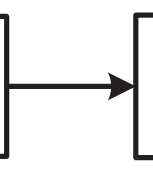

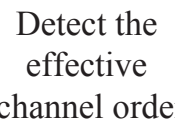
channel order

Figure 1 Block diagram of the proposed algorithm. 
where $\left\|\varepsilon_{L}\right\|_{2}=\lambda_{K+1}, \delta=\hat{\lambda}_{K}-\lambda_{K+1}$.

Thus,

$$
T \leq \frac{\lambda_{K+1}}{\delta}=\frac{\lambda_{K+1}}{\hat{\lambda}_{K}-\lambda_{K+1}}
$$

Then we have further that

$$
\hat{\lambda}_{K} \geq \lambda_{K}-\left\|\varepsilon_{L}\right\|_{2}=\lambda_{K}-\lambda_{K+1}
$$

If $\frac{\lambda_{K}}{\lambda_{K+1}} \geq 3$, then $\lambda>0$ and

$$
T \leq \frac{1}{\left(\lambda_{K} / \lambda_{K+1}\right)-2}
$$

Otherwise, our upper bound is equal to 1 . Therefore, we obtain

$$
f(K)=\left\{\begin{array}{cl}
\frac{1}{\left(\lambda_{K} / \lambda_{K+1}\right)-2}, & \frac{\lambda_{K}}{\lambda_{K+1}} \geq 3, \\
1, & \text { else. }
\end{array}\right.
$$

If $f(K) \ll 1$, then there is a gap between $\lambda_{K}$ and $\lambda_{K+1}$. The fact makes us consider the problem of decomposition into signal and noise subspaces. In this case, we obtain the numerical analysis arguments criterion as follows:

$$
\hat{K}=\arg \min _{K} f(K)
$$

Therefore, the effective channel order is detected by minimizing $f$. In addition, the derived criteria provides the "maximum stability" decomposition from the range space of the "over-modeled" data covariance matrix to the signal and noise subspaces. It is shown to be robust to variations in the signal-to-noise ratio (SNR) and the number of data samples. Of course, the bigger the noise is, the worse the estimated results are. In the simulation, it is assumed that the channel noise is small such that there is the big difference between the smallest signal eigenvalue and noise eigenvalue.

\subsection{Fourth-Order Cumulant Tensor}

In the following, we use the multilinearity property of the fourthorder cumulant tensor and construct the canonical model as follows:

$$
\mathcal{C}_{i j k l}^{\mathbf{x}}=\sum_{r=1}^{K} \kappa_{r} a_{i r} a_{j r}^{*} a_{k r}^{*} a_{l r}
$$

where $\mathcal{C}_{i j k l}^{\mathbf{x}}=\operatorname{Cum}\left\{\mathbf{x}, \mathbf{x}^{*}, \mathbf{x}^{*}, \mathbf{x}\right\} \in \mathbb{C}^{N \times N \times N \times N}$ is the fourth-order cumulant tensor of the observed signals, $\kappa_{r}$ represents the kurtosis of the rth source, and $a_{i r}, a_{j r}^{*}, a_{k r}^{*}, a_{l r}$ are factors of the canonical model. Combined with the cumulant tensor, factors of coined canonical decomposition are also the column vectors of the mixing matrix A.

It is assumed that the kurtosis values of source signals are known, then the decomposition model can be written as [29-32]

$$
\begin{aligned}
\overline{\mathbf{C}} & =\overline{\mathbf{K}} \times_{1} \mathbf{A}^{(1)} \times_{2} \mathbf{A}^{(2)} \times_{3} \mathbf{A}^{(3)} \times_{4} \mathbf{A}^{(4)}+\overline{\mathbf{E}} \\
& =\sum_{r=1}^{K} \kappa_{r}\left(a_{r}^{(1)} \circ a_{r}^{(2)} \circ a_{r}^{(3)} \circ a_{r}^{(4)}\right)+\overline{\mathbf{E}}
\end{aligned}
$$

where $\mathbf{A}^{(\circ)}=\left[a_{1}^{(\circ)}, a_{2}^{(\circ)} \cdots a_{N}^{(\circ)}\right]$ represents the tensor factor, 。= $1,2,3,4$, and $\overline{\mathbf{E}}$ represents the residual tensor. Within the mathematical framework, most signals are real values during the collection process. Based on the given condition, every array can be regarded as identical, which is represented by

$$
\mathbf{A}^{(1)}=\mathbf{A}^{(2)}=\mathbf{A}^{(3)}=\mathbf{A}^{(4)}
$$

In this paper, we use $I J \times K L$ matrix representation of $I \times J \times K \times L$ tensors as follows:

$$
\mathbf{C}^{\mathbf{x}}=\operatorname{mat}\left(\mathcal{C}_{i j k l}^{\mathbf{x}}\right) \Leftrightarrow(\mathbf{C})_{(i-1) J+j,(k-1) L+l}=\mathcal{C}_{i j k l}^{\mathbf{x}}
$$

where $\mathbf{C}^{\mathbf{x}} \in \mathbb{C}^{I J \times K L}$, and $\mathbf{C}^{\mathrm{s}}=\operatorname{diag}\left(\kappa_{1}, \cdots, \kappa_{K}\right) \in \mathbb{C}^{K \times K}$, i.e.,

$$
\mathbf{C}^{\mathbf{x}}=\left(\mathbf{A} \odot \mathbf{A}^{*}\right) \cdot \mathbf{C}^{\mathbf{s}} \cdot\left(\mathbf{A} \odot \mathbf{A}^{*}\right)^{H}
$$

Thus, the fourth-order statistics of the data are characterized by the $N^{2} \times N^{2}$ quadricovariance matrices, which are the representations of $N \times N \times N \times N$ tensors, i.e.,

$$
\mathbf{C}^{\mathbf{x}}=\operatorname{mat}\left(\mathcal{C}_{i j k l}^{\mathbf{x}}\right) \in \mathbb{C}^{N^{2} \times N^{2}}
$$

Then we calculate the eigenvalues of the matrix and combine numerical analysis arguments to estimate the effective channel order in the underdetermined case. It should be noted that if the number of source signals is much more than the number of sensors, fourth-order cumulant tensor method is not valid. For this reason, much more higher-order cumulant tensors need to be used.

\subsection{Sixth-Order Cumulant Tensor}

For the sake of convenience, we define the entries of the sixth-order cumulant tensor $\mathrm{C}_{\mathbf{x}} \in \mathbb{C}^{N \times N \times N \times N \times N \times N}$ of a random vector, and

$$
\mathcal{C}_{a b c, \mathbf{x}}^{d e f}=\operatorname{Cum}\left\{\mathbf{x}_{a}(t), \mathbf{x}_{b}(t), \mathbf{x}_{c}(t), \mathbf{x}_{d}(t)^{*}, \mathbf{x}_{e}(t)^{*}, \mathbf{x}_{f}(t)^{*}\right\}
$$

Such components can be ordered in the hexacovariance matrix:

$$
\forall 1 \leq a, b, c, d, e, f \leq N, \quad H_{N(N(a-1)+b-1)+c}^{N(N(d-1)+e-1)+f}=\mathcal{C}_{a b c, \mathbf{x}}^{d e f}
$$

Thanks to the multilinearity property of higher-order cumulant tensors, which implies:

$$
\mathbf{H}_{\mathbf{x}}=[\mathbf{A} \otimes \mathbf{A} \otimes \mathbf{A}] \mathbf{H}_{\mathbf{s}}[\mathbf{A} \otimes \mathbf{A} \otimes \mathbf{A}]^{H}
$$

where

$$
\mathbf{H}_{\mathbf{x}}=\operatorname{mat}\left(\mathcal{C}_{a b c, \mathbf{x}}^{d e f}\right) \in \mathbb{C}^{N^{3} \times N^{3}}
$$

and $\mathbf{H}_{\mathbf{s}} \in \mathbb{C}^{K^{3} \times K^{3}}$ denotes the hexacovariance matrices of $\mathbf{x}(t)$ and $\mathbf{s}(t)$, respectively. Additionally, $\mathbf{H}_{\mathbf{s}}$ is a diagonal matrix. We use the $N^{3} \times N^{3}$ matrix representations of $N \times N \times N \times N \times N \times N$ tensors and calculate the eigenvalues of the matrix $\mathbf{H}_{\mathbf{x}}$. Then, combining numerical analysis arguments to detect the effective channel order. By applying the sixth-order cumulant tensor, we can detect the effective channel order in the underdetermined case. It is capable of detecting up to $N^{2}-N+1$ sources from $N$ sensors. However, when the channel order is much larger, it is time consumption. This is also a question which is worth thoroughly pondering. The structure of the effective channel order determination is outlined in Figure 2. 


\section{SIMULATION RESULTS AND DISCUSSION}

In this section, we first test the proposed method to estimate the number of source signals in the overdetermined and underdetermined linear instantaneous mixture case. Meanwhile, information theoretic criteria are used to compare with our proposed method. Then the proposed method is used to detect the effective channel order in the convolutive mixture case. Finally, experiment results in terms of the probability of correct detection are shown as the performance measure. Meanwhile, we consider the computational complexity of the proposed algorithm. All the simulation experiments are conducted on a computer with Inter (R) Core (TM) i5-2537M CPU @ 1.40GHz, $4.00 \mathrm{~GB}$ memory under Windows 7 operational system and the programs are coded by Matlab R2015b installed in a personal computer.

\subsection{Source Number Estimation in Linear Instantaneous Mixture Case}

We consider the received signals with noise as follows:

$$
\mathbf{x}(t)=\mathbf{A s}(t)+\mathbf{n}(t)
$$

in which A denotes the linear mixing matrix, $\mathbf{n}(t)$ is Gaussian noise with zero mean and variance $\sigma^{2}$. Under the circumstances, the local covariances can be modeled as

$$
\mathbf{R}_{\mathbf{x}}(t)=\mathbf{A E}\left[\mathbf{s}(t) \mathbf{s}(t)^{H}\right] \mathbf{A}^{H}+\sigma^{2} \mathbf{I}
$$

In the simulation, we can estimate $\sigma^{2}$ using

$$
\hat{\sigma^{2}}=\min _{t=1, \ldots, M} \lambda_{\min }\left(\mathbf{R}_{\mathbf{x}}(t)\right)
$$

where $\lambda_{\min }(\cdot)$ denotes the magnitude-wise smallest eigenvalue. Additionally, we define the SNR as follows:

$$
\mathrm{SNR}=10 \log _{10} \frac{\frac{1}{M} \sum_{t=0}^{M-1} \mathbf{E}\|\mathbf{A s}(t)\|_{2}^{2}}{\mathbf{E}\|\mathbf{n}(t)\|_{2}^{2}}
$$

in which $M$ is the total number of samples.

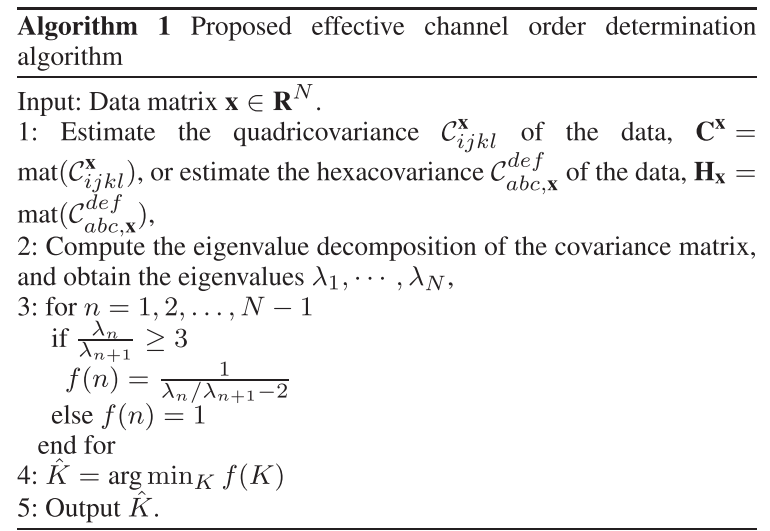

Figure 2 Proposed effective channel order determination algorithm.
In the first experiment, we estimate the number of source signals in the linear instantaneous mixture model, and consider an array with six sensors $N=6$, and four sources $K=4$. In the overdetermined case, using $M=500$ samples, and $\mathrm{SNR}=20 \mathrm{~dB}$. Meanwhile, the proposed method is compared with the classical information theoretic criteria. The simulation results are shown in Figure 3.

In the second experiment, we consider an array with six sensors $N=6$, and ten sources $K=10$, i.e., in the underdetermined case, using $M=500$ samples, and SNR $=20 \mathrm{~dB}$. The experiment results are shown in Figure 4.

Remark 1: According to Figure 3, it is shown that both information theoretic criteria and the proposed method demonstrate the effectiveness to detect the number of source signals in the overdetermined case. However, in the underdetermined case, information theoretic criteria are invalid, our proposed algorithm shows the effectiveness and superiority according to Figure 4.

\subsection{SIMO Convolutive Mixture Case}

In the third experiment, we consider a SIMO convolutive mixture case, and select six sensors $N=6$, one source signal $K=1$, the

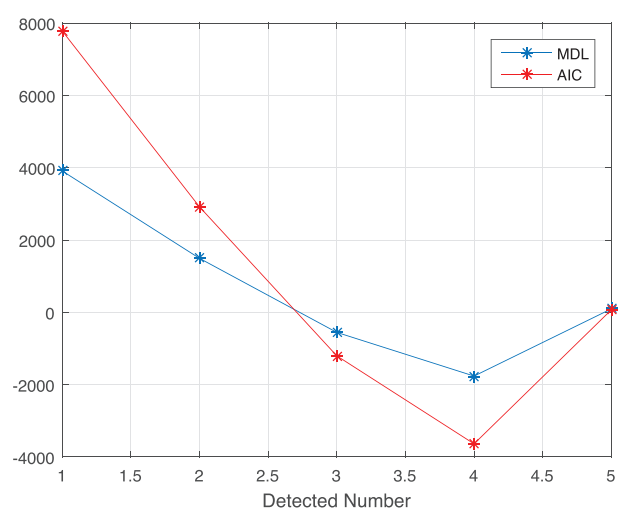

(a)

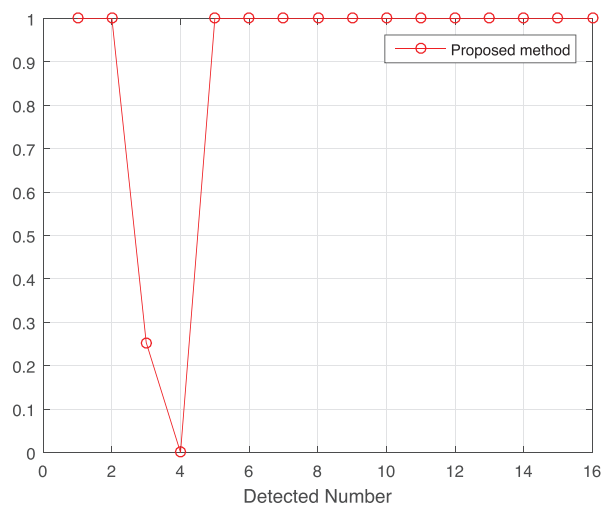

(b)

Figure 3 Detection of number of sources in overdetermined linear instantaneous mixture case with noise. (a) Information theoretic criteria.

(b) Proposed method. 


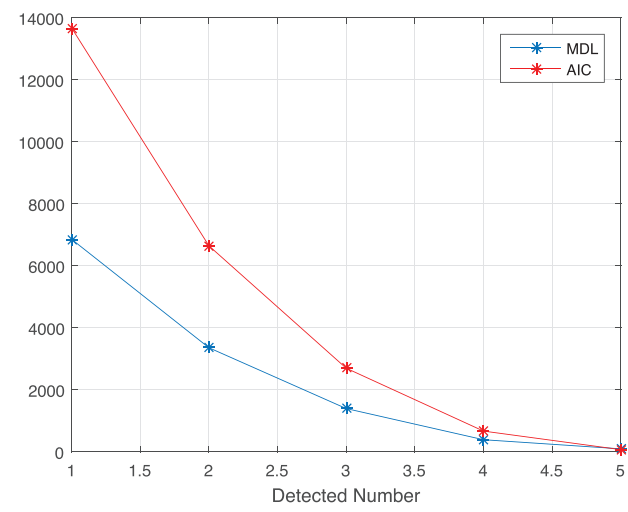

(a)

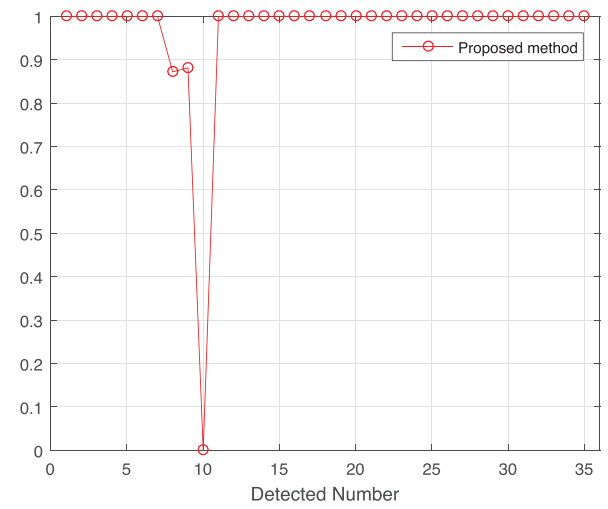

(b)

Figure 4 Detection of number of sources in underdetermined linear instantaneous mixture case with noise (a) Information theoretic criteria.

(b) Proposed method.

effective channel order is $L=15$, and the sample size $M=500$. The simulation results are shown in Figure 5.

\subsection{MIMO Convolutive Mixture Case}

In the fourth experiment, we consider a MIMO convolutive mixture case, and select five sensors $N=5$, four source signals $K=4$, the effective channel order is $L=2$, and the sample size $M=500$. The simulation results are shown in Figure 6.

\subsection{Underdetermined Convolutive Mixture Case}

In the fifth experiment, we consider an underdetermined convolutive mixture case, and select seven sensors $N=7$, eight source signals $K=8$, the effective channel order is $L=2$. The simulation results are shown in Figure 7.

Remark 2: According to Figure 5, it is shown that the effective channel order can be directly detected using the proposed algorithm. According to the simulation results in Figures 6 and 7, we obtain the relationship between the number of source signals and the effective channel order, i.e., $L=\hat{K} \div K$.

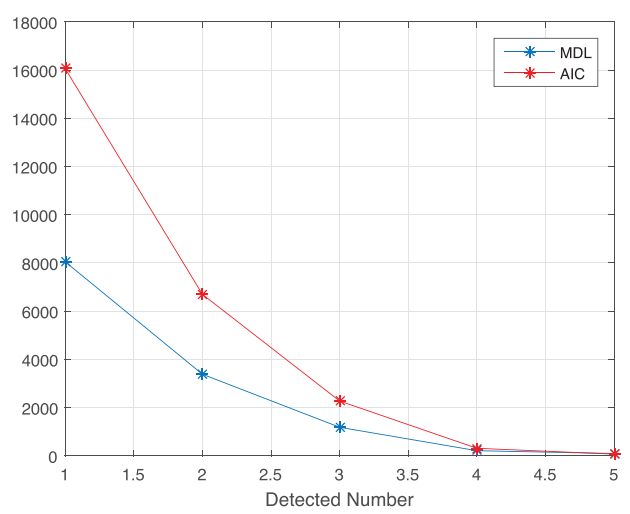

(a)

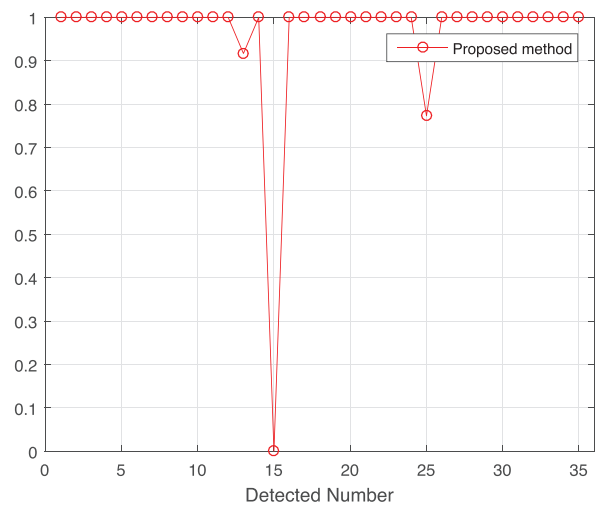

(b)

Figure 5 Detection of effective channel order in single-input multiple-output (SIMO) convolutive mixture case. (a) Information theoretic criteria.

(b) Proposed method.

\subsection{Results in Terms of the Probability of Correct Detection}

In the following, we conduct simulations in terms of the probability of the correct detection (PoD), i.e., $\operatorname{Pr}(\hat{K}=K)$, averaged over signal and noise realization. In the first case, we consider an array with seven sensors $N=7, K=1$, the effective channel order is $L=3$, and $\mathrm{SNR}=20 \mathrm{~dB}$. In the second case, we select seven sensors $N=7, K=1$, the effective channel order is $L=10$, and SNR $=20$ $\mathrm{dB}$. The simulation results are shown in Figure 8.

Remark 3: According to Figure 8, it is shown that PoDs of all algorithms increase monotonically with SNR and attain 1 at SNR = $20 \mathrm{~dB}$. Therefore, we select $\mathrm{SNR}=20 \mathrm{~dB}$ as the fixed value. However, when the effective channel order is larger than the number of sensors, the information theoretic criteria are invalid. In this case, the proposed algorithm has superior performance compared with information theoretic criteria.

\subsection{Computational Complexity of the Proposed Algorithm}

Finally, we consider the computational complexity of the proposed algorithm. For high-order cumulant tensor algorithm, one of the 


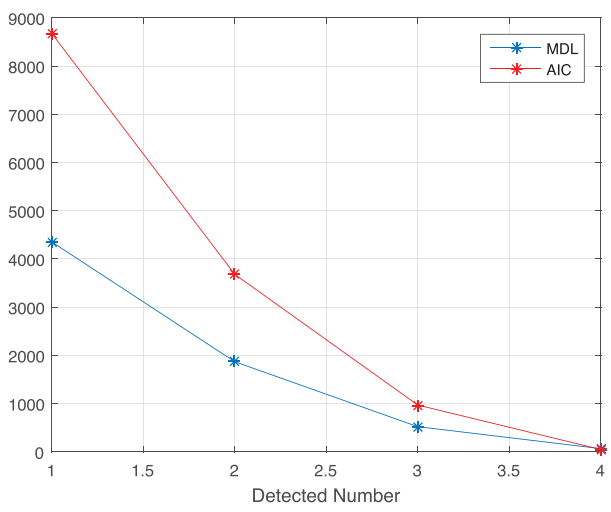

(a)

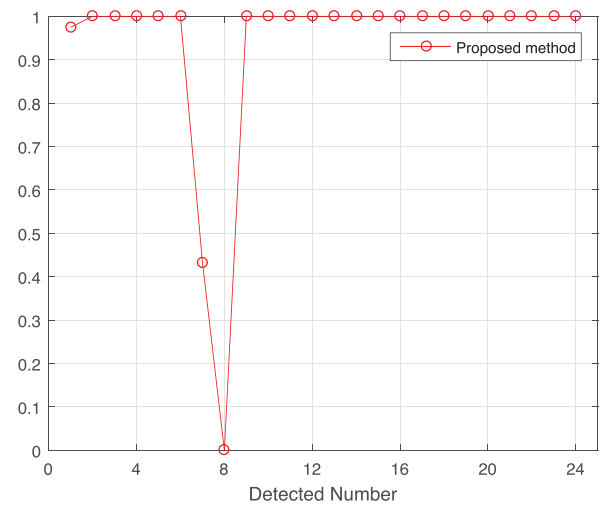

(b)

Figure 6 Detection of effective channel order in multiple-input multiple-output (MIMO) convolutive mixture case. (a) Information theoretic criteria. (b) Proposed method.

most time-consuming calculations is the matrix-matrix multiplication. For example, $\mathcal{C}_{i j k l}^{\mathbf{x}}$ in (13), whose computational complexity is $\boldsymbol{O}\left(N^{4}\right)$, which is controlled by the number of sensors $N$. In addition, we consider the running time of the proposed algorithm, and select the effective channel order is $L=30$ and the number of sensors is changed from 10 to 30. The results are shown in Figure 9. With the increasing of sensor number, it leads to time consumption. This will be an interesting issue for future research.

Additionally, we consider the comparisons between the fourthorder and sixth-order cumulant tensors. We select seven sensors $N=7$, one source signals $K=1$, the effective channel order is $L=20$. The simulation results are shown in Figure 10. It can be seen that the effective channel order can be detected correctly using the fourth-order and sixth-order cumulant tensors. However, compared with the computational complexity, we find that the computational complexity of sixth-order tensor is $\boldsymbol{O}\left(N^{6}\right)$, which is controlled by the number of sensors $N$. Therefore, it is time consumption.

\section{CONCLUSION AND FUTURE WORK}

In this paper, we provided an improved algorithm by integrating the numerical analysis arguments and higher-order cumulant

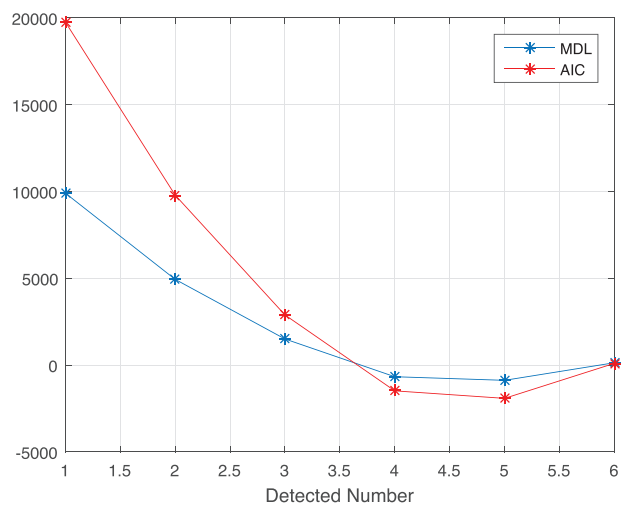

(a)

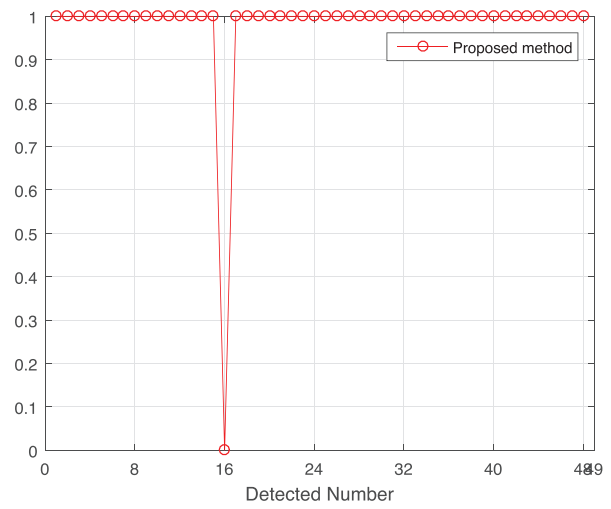

(b)

Figure 7 Detection of effective channel order in underdetermined convolutive mixture case.

(a) Information theoretic criteria. (b) Proposed method.

tensor to detect the number of source signals and the effective channel order in the underdetermined convolutive mixture case. Especially, in the SIMO case, the effective channel order can be estimated directly. Furthermore, we obtain the relationship between the number of source signals and the effective channel order based on the experimental results. Finally, numerous simulation experiments demonstrate the effectiveness and reasonableness of the proposed algorithm.

In addition, some further research directions need to be considered. First of all, it is worth noting that the higher effective channel order is, the more higher-order cumulant tensors need to be used. However, it is time consumption. Thus, much better algorithms need to be proposed to obtain the effective channel order, bringing to sufficiently good blind approximation of measured realworld microwave radio channels. Furthermore, effective channel order determination is an important issue in the convolutive blind source separation.

\section{CONFLICT OF INTEREST}

No Conflict of Interest. 


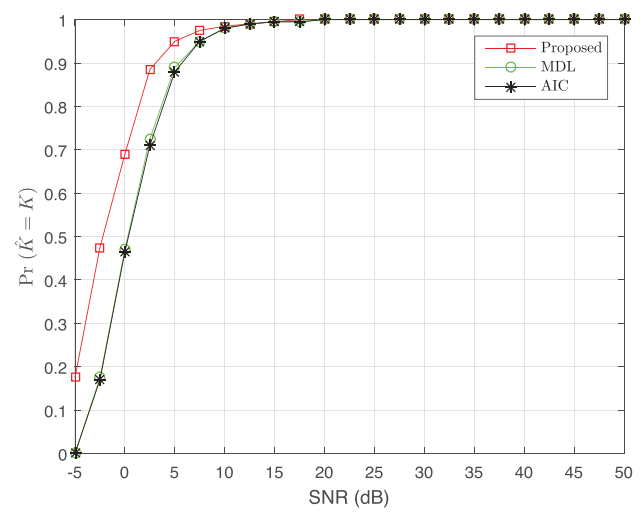

(a)

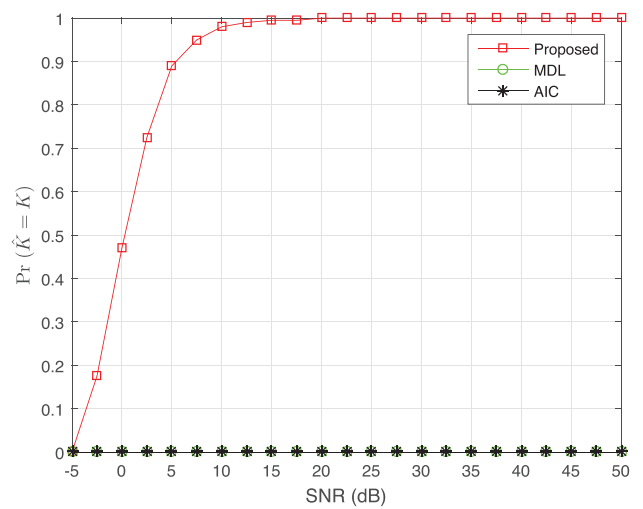

(b)

Figure 8 Results in terms of probability of the correct detection $(\mathrm{PoD})$ versus $(\mathrm{a})$ overdetermined convolutive mixture case, $(\mathrm{b})$ underdetermined convolutive mixture case.

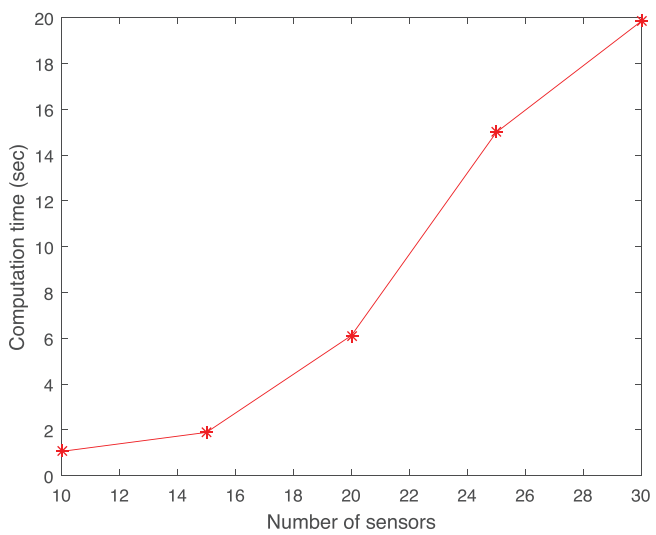

Figure 9 Running time of the proposed algorithm.

\section{AUTHORS' CONTRIBUTIONS}

Conceptualization, S.Y. Data curation, H.S. Funding acquisition, P.L. Methodology, S.Y. Project administration, J.C. Software, P.L. and S.H. Supervision, H.S. Validation, P.L., S.H. and H.S. Writing, original draft, S.Y. Writing, review and editing, S.Y. and P.L.

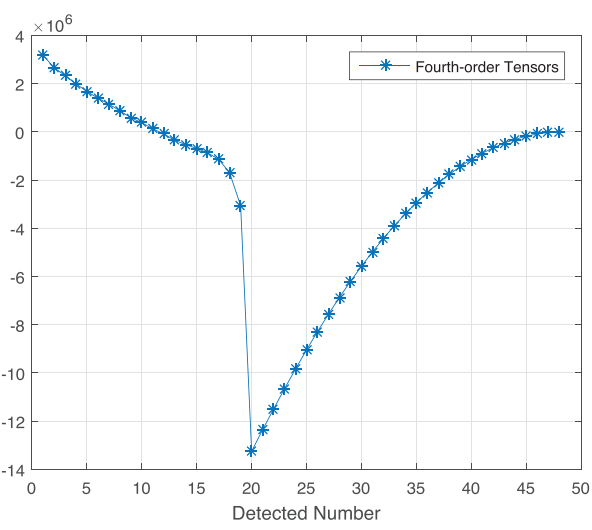

(a)

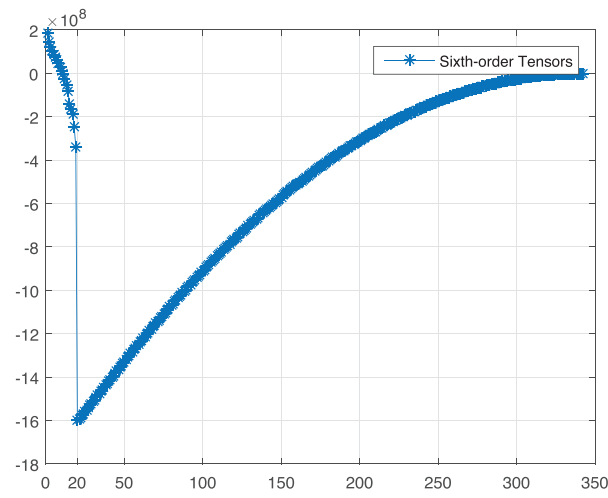

(b)

Figure 10 Detection of effective channel order in underdetermined convolutive mixture case.
(a) Fourth-order cumulant tensors.
(b) Sixth-order cumulant tensors.

\section{Funding Statement}

This research was funded in part by the National Natural Science Foundation of China under Grants 61773127, 61773128 and 61727810, the Science and Technology Program of Shaoguan City of China under Grant No. SK201644, the Natural Science Foundation of Guangdong Province of China under Grant 2016KQNCX156 and 2018A030307063, and Nature Science Foundation of Hebei Province under Grant E2016106018.

\section{REFERENCES}

[1] A.S. Lalos, C.G. Tsinos, K. Berberidis, Sparse subspace tracking techniques for adaptive blind channel identification in ofdm systems, in IEEE International Conference on Acoustics, Speech and Signal Processing (ICASSP), Kyoto, 2013, vol. 5, pp. 3185-3188.

[2] S. Vanvaerenbergh, J. Va, I. Santamara, Blind identification of simo wiener systems based on kernel canonical correlation analysis, IEEE Trans. Signal Process. 61 (2013), 2219-2230.

[3] J.J. Yang, H.L. Lin, Blind identification of the underdetermined mixing matrix based on k-weighted hyperline clustering, Neurocomputing. 149 (2015), 483-489. 
[4] D. Nion, K.N. Mokios, N.D. Sidiropoulos, A. Potamianos, Batch and adaptive parafac-based blind separation of convolutive speech mixtures, IEEE Trans. Audio Speech Lang. Process. 18 (2010), 1193-1207.

[5] S. Xie, L. Yang, J.M. Yang, G. Zhou, Y. Xiang, Time-frequency approach to underdetermined blind source separation, IEEE Trans. Neural Netw. Learn. Syst. 23 (2012), 306-316.

[6] Y. Xie, K. Xie, S. Xie, Underdetermined convolutive blind separation of sources integrating tensor factorization and expectation maximization, Digital Signal Process. 8 (2019), $145-154$.

[7] Y. Xie, K. Xie, J. Yang, Z. Wu, S. Xie, Underdetermined reverberant audio-source separation through improved expectationcmaximization algorithm, Circuits Syst. Signal Process. 38 (2019), 2877-2889.

[8] Y. Xie, K. Xie, J. Yang, S. Xie, Underdetermined blind source separation combining tensor decomposition and nonnegative matrix factorization, Symmetry. 10(2018), 521.

[9] M. Wax, T. Kailath, Detection of signals by information theoretic criteria, IEEE Trans. Acoustics Speech Signal Process. 33 (1985), 387-392.

[10] K.M. Wong, Q.T. Zhang, J.P. Reilly, P.C. Yip, On information theoretic criteria for determining the number of signals in high resolution array processing, IEEE Trans. Acoustics Speech Signal Process. 38 (1990), 1959-1971.

[11] H. Chen, Estimating the dimension of a model, Asia-Pac. J. Risk Insur. 6 (1978), 461-464.

[12] S. Kqnishi, G. Kitagawa, Generalised information criteria in model selection, Biometrika. 83 (1996), 875-890.

[13] H. Akaike, A New Look at the Statistical Model Identification, New York, 1974.

[14] K. Liu, J.P.C.L. Da Costa, H. Cheung So, L. Huang, J. Ye, Detection of number of components in candecomp/parafac models via minimum description length, Digital Signal Process. 5 (2016), $110-123$

[15] R.R. Nadakuditi, A. Edelman, Sample eigenvalue based detection of high-dimensional signals in white noise using relatively few samples, IEEE Trans. Signal Process. 56 (2008), 2625-2638.

[16] S. Kritchman, B. Nadler, Non-parametric detection of the number of signals: hypothesis testing and random matrix theory, IEEE Trans. Signal Process. 57 (2009), 3930-3941.

[17] V.Y.F. Tan, C. Fevotte, Automatic relevance determination in nonnegative matrix factorization with the -divergence, IEEE Trans. Pattern Anal. Mach. Intell. 35 (2013), 1592-1605.

[18] Z. He, A. Cichocki, S. Xie, K. Choi, Detecting the number of clusters in n-way probabilistic clustering, IEEE Trans. Pattern Anal. Mach. Intell. 32 (2010), 2006-2021.
[19] Y. Xie, K. Xie, S. Xie, Underdetermined blind source separation for heart sound using higher-order statistics and sparse representation, IEEE Access. 7 (2019), 87606-87616.

[20] A.P. Liavas, P.A. Regalia, J.P. Delmas, Blind channel approximation: effective channel order determination, IEEE Trans. Signal Process. 47 (1999), 3336-3344.

[21] A.P. Liavas, P.A. Regalia, J.P. Delmas, Robustness of least-squares and subspace methods for blind channel identification/equalization with respect to effective channel undermodeling/overmodeling, IEEE Trans. Signal Process. 47 (1999), 1636-1645.

[22] L. Albera, A. Ferrol, P. Comon, P. Chevalier, Sixth order blind identification of underdetermined mixtures (birth) of sources, in International Symposium on Independent Component Analysis and Blind Signal Separation, Nara, 2003, vol. 4, pp. 909-914.

[23] A. Ferreol, L. Albera, P. Chevalier, Fourth-order blind identification of underdetermined mixtures of sources (fobium), IEEE Trans. Signal Process. 53 (2005), 1640-1653.

[24] L. De Lathauwer, J. Castaing, J.F. Cardoso, Fourth-order cumulant-based blind identification of underdetermined mixtures, IEEE Trans. Signal Process. 55 (2007), 2965-2973.

[25] Y. Xie, K. Xie, S. Xie, Source number estimation and effective channel order determination based on higher-order tensors, Circuits Syst. Signal Process. 2(2019), 1-16.

[26] T. Tatsuya, N. Lee, A. Cichocki, Robust multilinear tensor rank estimation using higher order singular value decomposition and information criteria, IEEE Trans. Signal Process. 65 (2016), 1196-1206.

[27] F. Roemer, M. Haardt, G. Del Galdo, Analytical performance assessment of multi-dimensional matrix- and tensor-based esprit-type algorithms, IEEE Trans. Signal Process. 62 (2014), 2611-2625.

[28] M. Haardt, F. Roemer, G. Del Galdo, Higher-order svd-based subspace estimation to improve the parameter estimation accuracy in multidimensional harmonic retrieval problems, IEEE Trans. Signal Process. 56 (2008), 3198-3213.

[29] S. Ge, J. Han, M. Han, Nonnegative mixture for underdetermined blind source separation based on a tensor algorithm, Circuits Syst. Signal Process. 34 (2015), 2935-2950.

[30] T.G. Kolda, B.W. Bader, Tensor decompositions and applications, Siam Rev. 51 (2009), 455-500.

[31] A. Cichocki, D.P. Mandic, A.H. Phan, C.F. Caiafa, G. Zhou, Q. Zhao, L. Lathauwer, Tensor decompositions for signal processing applications from two-way to multiway component analysis, IEEE Signal Process. Mag. 32 (2015), 145-163.

[32] C.I. Kanatsoulis, X. Fu, N.D. Sidiropoulos, W. Ma, Hyperspectral super-resolution: a coupled tensor factorization approach, IEEE Trans. Signal Process. 66 (2018), 6503-6517. 